\title{
EFFICIENT PHOTOEMISSION FROM ROBUST FERROELECTRIC CERAMICS
}

\author{
I. Boscolo, University and INFN, Via Celoria 16, 20133 Milano, Italy \\ M. Castellano, L. Catani, M. Ferrario, F. Tazzioli, INFN-LNF, \\ Via E. Fermi, 00044 Frascati - Roma, Italy \\ L. Giannessi, ENEA-CRE, Via E. Fermi, 00044 Frascati - Roma, Italy
}

\begin{abstract}
Experimental results on photoemission by ferroelectric ceramic disks, with a possible interpretation, are presented. Two types of lead zirconate titanate lanthanum doped, PLZT, ceramics have been used for tests. 25 ps light pulses of 532 and $355 \mathrm{~nm}$ were used for excitation. The intensity ranged within the interval $0.1-3 \mathrm{GW} / \mathrm{cm}^{2}$. The upper limit of the intensity was established by the damage threshold tested by the onset of ion emission. At low value of the intensity the yield was comparable at the two wavelengths. At the highest intensity of green light the emitted charge was $1 \mathrm{nC}$ per $10 \mathrm{~mm}^{2}$, but it was limited by the space charge effect. In fact, the applied field was only $20 \mathrm{kV} / \mathrm{cm}$, allowed both by the mechanical design of the apparatus and the poor vacuum, $10^{-4}$ mbar. No surface processing was required. The measurement of the electron pulse length under way.
\end{abstract}

\section{INTRODUCTION}

Lead zirconate titanate lanthanum doped (referred as PLZT) ferroelectric ceramic showed interesting properties as photoemitter material $[1,2]$ : this type of photo-cathode showed an emissivity higher than that of metals, they were able to emit at any photon energy from green to UV and they were very robust, they did not need any processing and, furthermore, they did not require high vacuum condition.

An experimental program has been set at the LNF (Lab Nationali Frascati-Roma) based on the fact that the properties of this material can be determined simply changing the compositional percentage and changing the polarization state. The physical state of the surface is strongly changed by prepoling and by setting a polarization state. In particular, it seems possible to set a polarization state such that the surface electrons are acted on by a repulsive force, or, alternatively, it is possible to set at the surface a very dense sheet of electrons[3].

The sketch of the experimental setup is shown in fig.1. Two incidence angles were used: $60^{\circ}$ and $0^{\circ}$. In the latter case a hollow Faraday cup with a front grid was used. No variation of the yield was measured for the two configurations.

The experimental program started with the material good for emission with electric excitation [4, 5], that is PLZT $8 / 65 / 35$ and $4 / 95 / 5$, where the numbers refer to lanthanum

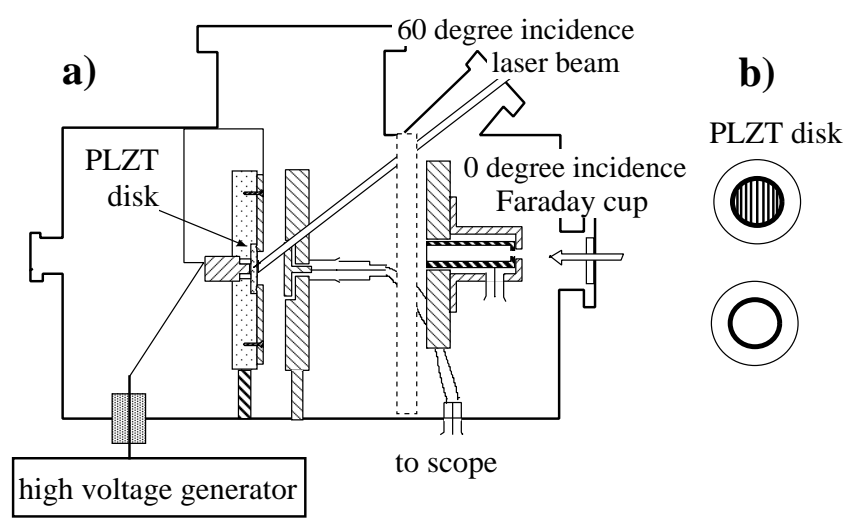

Figure 1: a) Sketch of the experimental apparatus used in the photoemission experiments: the two used Faraday cups are shown for simplicity on the same figure (one behind the other); they are interchanged depending on the incidence angle. b) Sketch of the cathode with the two front electrodes used in tests. The passively mode locked Nd-YAG laser provides some $\mathrm{mJ}$ of light at $\lambda=532 \mathrm{~nm}$ for a pulse length of $25 \mathrm{ps}$. The illuminated area was about $10 \mathrm{~mm}^{2}$

(in relation to lead), zirconium and titanium relative atom percentage. Samples without and with prepoling, at room temperature, were tested. Ceramic $8 / 65 / 35$ is in ferroelectric phase, while $4 / 95 / 5$ is in antiferroelectric phase. These materials have a high density of defects whose activation energy is about $1 \mathrm{eV}$ [6]. The cathodes are disks of $16 \mathrm{~mm}$ diameter and $1 \mathrm{~mm}$ thickness, coated by a uniform metallic film at the back surface and by either an external ring or a grating at the front surface, see fig. $1 \mathrm{~b}$ ). The best results came with the ring front electrode and 8/65/35 unprepoled samples.

\section{EXPERIMENTAL RESULTS}

The emission in the log-log diagram from a PLZT 8/65/35 is shown in figs. 2 and 3. The emission was limited by the space charge effect in the case of green light shining, it was not in the case of violet light shining.

The damage threshold has been checked reversing the direction of the accelerating field. In fig. 4 it is shown that the energy at which the ion emission starts is farther than the beginning of the space charge effect.

From figs. 2 and 3 we notice: a threshold with green light, a yield of an angular coefficient nearby 4 for green light and nearby 3 for violet light. Extrapolating with an 


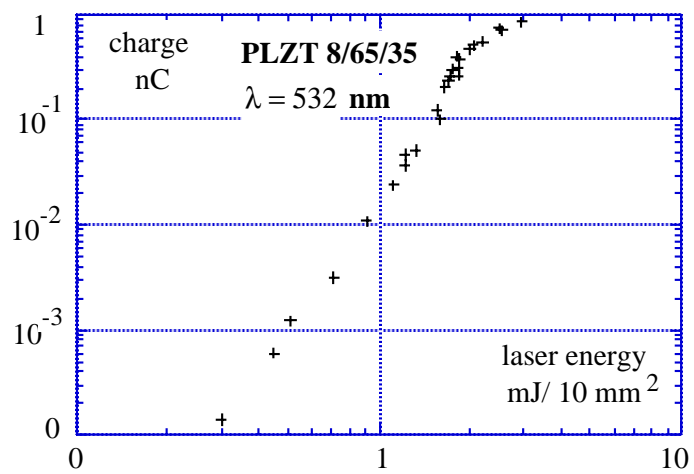

Figure 2: Emitted charge versus laser energy for PLZT $8 / 65 / 35$ shined with green light in log-log frame. The line fitting the points scales as $Q \alpha I^{4}$.

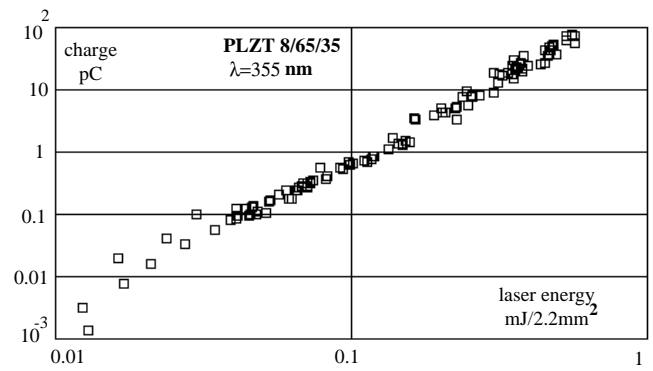

Figure 3: Emitted charge versus laser energy for PLZT $8 / 65 / 35$ shined with violet light in $\log$-log frame. The slope of the line is $Q \alpha I^{3}$.

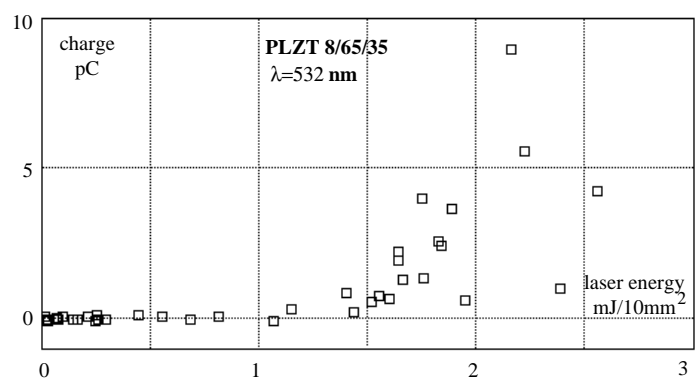

Figure 4: Starting of emission of ions versus laser energy with green light.

accelerating field high enough to avoid saturation effects, the emitted charge at $3 \mathrm{~mJ}$ of laser light would be $2 \mathrm{nC}$. The value of quantum efficiency results around $10^{-6}$ with both wavelengths.

With violet light we could shine with an energy up to 0.5 mJ only.

The experiments with the antiferroelectric material 4/95/5 and hard ferroelectric material lead titanate (PT) gave a much lower yield. The 4/95/5 material showed quite a high enhancement of the yield increasing the accelerating field in the gap as shown in fig. 5. It is notable that this antiferroelectric material shows an hysteretic behavior as function of the applied voltage.

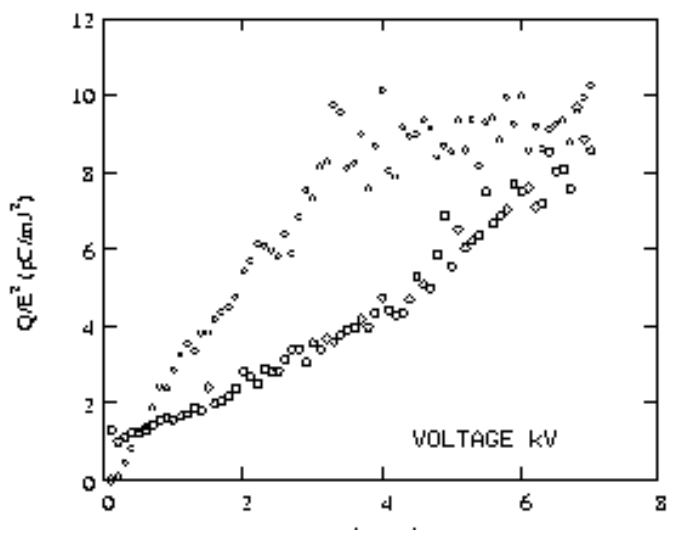

Figure 5: Emitted charge versus laser energy: the lower points are obtained at $7 \mathrm{kV}$ of applied voltage, while the points of the upper curve are obtained in succession but after having reduced the voltage to $3.5 \mathrm{kV}$. The hysteretic behavior was not observed keeping constant the voltage.

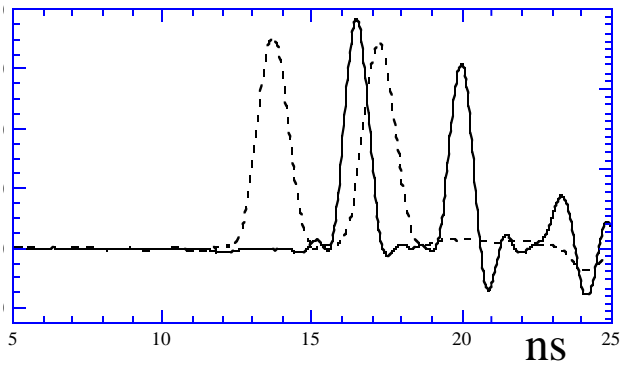

Figure 6: Succession of two electron pulses 2 ns apart

All the tested ceramics had the scaling law $Q \alpha I^{2}$ at relatively low light flux for both wavelength.

We have tested the emission with two light pulses separated by 2 ns 6 . The two emissions are substantially stable. The system seems able to provide pulse trains with nanosecond time separation.

The yield of the prepoled samples was higher than that of the unprepoled ones, but the damage threshold was considerably lower. They showed an emission law which was a bit faster than the two photon emission, but the relatively low damage threshold did not allow an efficient emission.

\section{DISCUSSION}

The two main characteristics of the strong emission are: a very low emission up to a laser intensity of about 0.5 $\mathrm{GW} / \mathrm{cm}^{2}$ and the high non-linearity starting from that point. In addition to this, the other notable fact is the change of the operational regime for the PLZT 4/95/5 sample when it is immersed in a relatively high electric field.

The energy diagram of the material shows a trap level at $1 \mathrm{eV}$ from the conduction band and has an estimated electron affinity of $3 \mathrm{eV}$. The electron affinity $\mathrm{E}_{a}$ is not well defined because the surface state is un-defined: is like a 
patchwork of pieces with different physical characteristics, which range from insulating to metallic [8]. A value of the potential barrier greater than $4 \mathrm{eV}$ is a fairly crude approximation. The quadratic power law of the emission at both photon energy of 2.3 and $3.4 \mathrm{eV}$ is congruent with the energy diagram.

Furthermore, our disk is immersed in the electric field applied through the diode gap, hence a counter field is created by the induced polarization. When the crystal is polarized, there is a band bending at the surfaces with a potential well for electrons at the positive side of the polarization.

The generalized Fowler-Dubrige theory [7] cannot explain these results. The emission at $2.3 \mathrm{eV}$ and its nonlinearity with a power equal or greater than 4 would envision the anomalous heating regime [9], cooperating with the Auger effect [3]. More generally, we should have the concurrence of different contributions: one and two-photon emission, thermally assisted and Auger emission.

The increase of the emission of 4/95/5 sample as a function of the applied field, together with its hysteretic behavior of fig. 5 tells that the polarization is very important: when the polarization builds up in the sample, the emission steps up, then the sample remains polarized when the electric field is reduced because of the hysteresis loop. The experiment with PT material says that the polarization by itself is not sufficient for obtaining strong emission, but a strong doping, that is a large number of defects, must be also present.

Assuming that the electron pulse length is strictly correlated to the light pulse length, that is $\approx 25 \mathrm{ps}$, since the illuminated area is about $10 \mathrm{~mm}^{2}$, the current density would be higher than $1 \mathrm{kA} / \mathrm{cm}^{2}$.

\section{CONCLUSIONS.}

A new very efficient configuration for ferroelectric photocathodes has been investigated. We got $1 \mathrm{nC}$ level of emission only because the charge was limited by space charge effect. Since the damage threshold of a ceramic is relatively high, a large amount of extracted charge can be foreseen.

The emission has shown to be very sensitive to the sample polarization. This fact allows to foresee a large enhancement of the quantum efficiency just increasing the polarization. This polarization increasing occurs naturally with the high electric field that are applied in electron guns.

The characteristics of these cathodes, are: a)strong robustness, they work in any kind of vacuum showing a long life; b) they do not require any particular processing; c)they can be operated with green light. In the next future the extracted electron beam will be characterized in terms of time structure. If the electron pulse duration is strictly related to the laser pulse duration, these cathodes promise to deliver current densities larger than $1 \mathrm{KA} / \mathrm{cm}^{2}$ and to be valid competitors of both metallic and alkali cathodes.

\section{ACKNOWLEDGEMENTS}

We recognize the technical support given by $\mathrm{R}$. Sorchetti and L. Cacciotti.

\section{REFERENCES}

[1] K. Geissler, H. Gundel, H. Riege, J. Handerek, Appl. Phys. Lett. 56, 895, 1990.

[2] K. Geissler, A. Meineke, H., Riege, S. DE Silvestri, N. Nisoli, O. Svelto, I. Boscolo, J. Handerek, Nucl. Instrum. Meth. Phys. Res.A 372, 567-571,1996

[3] G. Benedek, I. Boscolo, Appl. Phys. Lett. 72, 522, 1998.

[4] G. Benedek, I. Boscolo, J. Handerek, A. Moscatelli, A. Scurati, J. Appl. Phys, 83,2776,1998.

[5] I. Boscolo et al. Appl. Phys. Lett. 74,859,1999.

[6] H. Gundel, J. Handerek, H. Riege, E.J.N. Wilson and K. Zioutas, Ferroelectrics 109,137,1990.

[7] J.H. Bechtel, W.L. Smith, N. Bloembergen, Phys. Rev. B. 15, 4557, 1977.

[8] K. Szot, W. Speier, J. Herion, Ch. Freiburg, Appl. Phys. A $64,55,1997$.

[9] J. P. Girardeau-Montaut, C. Girardeau-Montaut, Phys. Rev. $51,13560,1995$. 